\title{
Editorial \\ What do biomarkers tell us about the pathogenesis of ankylosing spondylitis?
}

Walter P Maksymowych

Department of Medicine, University of Alberta, Edmonton, Alberta T6G 2S2 Canada

Corresponding author: Walter P Maksymowych, walter.maksymowych@ualberta.ca

See related research article by Appel et al., http://arthritis-research.com/content/10/5/R125

Published: 7 January 2009

This article is online at http://arthritis-research.com/content/11/1/101

(c) 2009 BioMed Central Ltd

\begin{abstract}
Biomarkers may provide information that promotes understanding of prognosis, disease activity, and pathogenesis in ankylosing spondylitis. Biomarkers reflecting disease activity (metalloproteinase-3) and inflammatory lesions on magnetic resonance imaging predict new bone formation and are ameliorated by antitumor necrosis factor therapy, yet this treatment may not prevent new bone formation. Moreover, elevated levels of biomarkers reflecting tissue repair (bone-specific alkaline phosphatase) posttreatment together with magnetic resonance imaging indicates such treatment may even promote repair through new bone formation. Tumor necrosis factor regulation of Dickkopf-1 may constitute a molecular brake that controls osteoblastogenesis through wingless and bone morphogenetic proteins in an established inflammatory lesion in ankylosing spondylitis.
\end{abstract}

Appel and colleagues [1] have studied the link between inflammation, destruction, and repair in ankylosing spondylitis (AS) by analyzing serological biomarkers thought to reflect these processes. How does this advance our understanding and assessment of AS? First, biomarkers may provide information that is of prognostic value, particularly if sufficiently validated to be considered surrogates for structural damage end points. This constitutes a major priority for investigation in AS because of the generally slow rate of progression of radiographic change [2]. Such a biomarker could then be used to enrich for rapid progressors in trials of disease modifying therapies. To date, one study has shown that serum metalloproteinase (MMP) 3 may predict radiographic progression in AS [3].

Second, biomarkers may be of value in the objective assessment of disease activity. This is again a priority in AS because measures of disease activity are limited to patient selfreported questionnaires and standard laboratory measures,
Arthritis Research \& Therapy 2009, 11:101 (doi:10.1186/ar2565)

such as acute phase reactants, lack sensitivity and specificity. Moreover, although magnetic resonance imaging (MRI) demonstrates inflammatory lesions that can be reliably quantified, it is not readily available or feasible for prospective study. In peripheral joints, there is evidence that MMP3 reflects a histopathological grade of inflammation in patients with spondylarthropathy [4]. Such direct investigation of the relationship between biomarker levels and inflammation at the tissue level is clearly not feasible in the spine and limited analysis has focused on associations with MRI scores for spinal inflammation. Significant correlations have been reported with interleukin- 6 and vasoactive epidermal growth factor (VEGF) but not with serum MMP3 [5,6].

Third, biomarkers may clarify our understanding of the pathophysiology of disease. Unlike rheumatoid arthritis, there is still no formal proof that inflammation is necessarily associated with radiographic change in AS. It has even been suggested that inflammation and new bone formation are uncoupled [7]. Moreover, comparison of radiographic progression in AS patients receiving anti-tumor necrosis factor (anti-TNF) therapy with those receiving standard therapy has not revealed any significant difference $[8,9]$. Prospective MRI examination even shows that while inflammatory changes in the spine predict the development of new syndesmophytes, complete resolution of inflammation at a vertebral corner following the institution of anti-TNF therapy does not prevent the development of new syndesmophytes [10]. On the contrary, it appears that new syndesmophytes are even more likely to develop at those vertebral corners demonstrating resolution of inflammation.

Appel and colleagues assessed the impact of treatment with adalimumab on serum MMP3, VEGF, and bone specific alka-

AS = ankylosing spondylitis; BALP = bone specific alkaline phosphatase; BMP, bone morphogenetic protein; DKK = Dickkopf; MMP = metalloproteinase; $\mathrm{MRI}=$ magnetic resonance imaging; TNF = tumor necrosis factor; VEGF = vasoactive epidermal growth factor. 
line phosphatase (BALP) over a period of 2 years and compared this to biomarker levels in patients followed in an observational cohort, GESPIC. While no change in biomarkers was evident in GESPIC patients over 2 years, a significant reduction in MMP3 and VEGF was observed that correlated with a change in $\mathrm{C}$ reactive protein in adalimumab treated patients. Conversely, a significant increase in BALP was noted that correlated inversely with a change in MMP3. These observations support the findings from several other studies with anti-TNF agents [5,6] and are consistent with observations from MRI studies where resolution of inflammation with anti-TNF therapy is followed by new bone formation [10].

How might these findings be explained at a molecular level? Recent work has shown that TNF alpha stimulates expression of Dickkopf (DKK)-1, a major regulator of joint remodeling through its suppression of signaling by wingless proteins (Wnt), which are key mediators of osteoblastogenesis [11]. Moreover, regulation of Wnt signaling by DKK-1 and - 2 regulates osteoblast differentiation induced by bone morphogenetic protein (BMP)-2 [12]. So once inflammation is allowed to resolve through pharmacological suppression of TNF alpha, this may allow signaling through Wnt and other growth factors such as BMP-2 to induce new bone formation. TNF alpha may be viewed as a molecular brake on new bone formation acting through DKK-1, but this then begs the question as to whether anti-TNF therapy might in fact accelerate new bone formation in patients with AS and why this was not observed in clinical trials. It is important to address this in the context of the histopathological evolution of an inflammatory lesion in the spine. Early lesions have features of synovial and subchondral marrow inflammation that is followed by erosion of subchondral bone, formation of woven bone and metaplastic cartilage, and finally synthesis of endochondral bone by osteoblasts. In established AS, each patient is likely to have several spinal lesions at different stages of evolution and it may be possible that very early lesions resolve with anti-TNF therapy prior to the induction of reparative changes while accelerated reparation is observed with anti-TNF therapy in the more mature inflammatory lesions where reparation is already underway. The overall outcome for the individual patient is then little changed at the level of the entire spine. Lack of impact of adalimumab on BALP in pre-radiographic disease is consistent with this hypothesis.

This raises the concept of a window of opportunity for disease modification in AS using anti-TNF therapy. New trials of anti-TNF therapies in patients presenting early in their disease course prior to the appearance of significant repair in the spine are now a high priority.

\section{Competing interests}

WPM has received honoraria and/or research grants from Amgen/Wyeth, Schering Canada, Abbott Labs.

\section{References}

1. Appel H, Janssen L, Listing J, Heydrich R, Rudwaleit M, Sieper J: Serum levels of biomarkers of bone and cartilage destruction and new bone formation in different cohorts of patients with axial spondyloarthritis with and without tumor necrosis factor-alpha blocker treatment. Arthritis Res Ther 2008, 10: R125.

2. Wanders AJ, Landewé RB, Spoorenberg A, Dougados M, van der Linden S, Mielants $H$, van der Tempel $H$, van der Heijde DM: What is the most appropriate radiologic scoring method for ankylosing spondylitis? A comparison of the available methods based on the Outcome Measures in Rheumatology Clinical Trials filter. Arthritis Rheum 2004, 50:2622-2632.

3. Maksymowych WP, Landewé R, Conner-Spady B, Dougados M, Mielants $\mathrm{H}$, van der Tempel $\mathrm{H}$, Poole AR, Wang $\mathrm{N}$, van der Heijde $D$ : Serum matrix metalloproteinase 3 is an independent predictor of structural damage progression in patients with ankylosing spondylitis. Arthritis Rheum 2007, 56:1846-1853.

4. Vandooren B, Kruithof E, Yu DT, Rihl M, Gu J, De Rycke L, Van Den Bosch F, Veys EM, De Keyser F, Baeten D: Involvement of matrix metalloproteinases and their inhibitors in peripheral synovitis and down-regulation by tumor necrosis factor alpha blockade in spondylarthropathy. Arthritis Rheum 2004, 50: 2942-2953.

5. Visvanathan S, Wagner C, Marini JC, Baker D, Gathany T, Han J, van der Heijde D, Braun J: Inflammatory biomarkers, disease activity and spinal disease measures in patients with ankylosing spondylitis after treatment with infliximab. Ann Rheum Dis 2008, 67:511-517.

6. Maksymowych WP, Rahman P, Shojania K, Olszynski WP, Thomson GTD, Ballal S, Wong RL, Inman RD: Beneficial effects of Adalimumab on biomarkers reflecting structural damage in patients with ankylosing spondylitis. J Rheumatol 2008, 35: 2030-2037.

7. Lories RJ, Derese I, de Bari C, Luyten FP: Evidence for uncoupling of inflammation and joint remodeling in a mouse model of spondylarthritis. Arthritis Rheum 2007, 56:489-497.

8. van der Heijde $D$, Landewé $R$, Baraliakos $X$, Houben $H$, van Tubergen A, Williamson P, Xu W, Baker D, Goldstein N, Braun J: Ankylosing Spondylitis Study for the Evaluation of Recombinant Infliximab Therapy Study Group: Radiographic findings following two years of infliximab therapy in patients with ankylosing spondylitis. Arthritis Rheum 2008, 58:3063-3070.

9. van der Heijde D, Landewé R, Einstein S, Ory P, Vosse D, Ni L, Lin SL, Tsuji W, Davis JC Jr: Radiographic progression of ankylosing spondylitis after up to two years of treatment with etanercept. Arthritis Rheum 2008, 58:1324-1331.

10. Maksymowych WP, Chiowchanwisawakit P, Clare T, Pedersen S, Ostergaard M, Lambert RGW: Inflammatory lesions of the spine on MRI predict the development of new syndesmophytes in ankylosing spondylitis: Evidence for coupling between inflammation and new bone formation. Arthritis Rheum, in press.

11. Diarra D, Stolina M, Polzer K, Zwerina J, Ominsky MS, Dwyer D, Korb A, Smolen J, Hoffmann M, Scheinecker C, van der Heide D, Landewe R, Lacey D, Richards WG, Schett G: Dickkopf-1 is a master regulator of joint remodeling. Nat Med 2007, 13:156163.

12. Fujita K, Janz S: Attenuation of WNT signaling by DKK-1 and $\mathbf{- 2}$ regulates $B M P 2-i n d u c e d$ osteoblast differentiation and expression of OPG, RANKL and M-CSF. Mol Cancer 2007, 6:71. 